\title{
Estudo da Heterogeneidade das Variâncias para as Características Produtivas de Rebanhos da Raça Holandesa no Rio Grande do Sul ${ }^{1}$
}

\author{
Angelo Elias Marion², Paulo Roberto Nogara Rorato ${ }^{3}$, Gilka Barbosa Ferreira ${ }^{3}$, Dionéia Magda \\ F. Everling ${ }^{2}$, Henrique Dorneles Fernandes ${ }^{4}$
}

\begin{abstract}
RESUMO - Com os objetivos de estudar a heterogeneidade das variâncias e estimar os coeficientes de herdabilidade em diferentes níveis de produção de leite dos rebanhos e em diferentes classes de desvio-padrão, foram analisados os registros de produção de 13.053 lactações de 6.828 vacas da raça Holandesa, filhas de 811 touros, distribuídas em 78 rebanhos no estado do Rio Grande do Sul, no período de 1988 a 1997. Os rebanhos foram agrupados pelo nível de produção e pelo desvio padrão da produção de leite, aos 305 dias, em três níveis (baixo, médio e alto). Os componentes de variância foram obtidos pelo método da Máxima Verossimilhança Restrita, utilizando o programa MTDFREML e um modelo animal misto incluindo os seguintes efeitos fixos: classe de idade, grupo genético, rebanho dentro de núcleo e ano-época de parto e os aleatórios do animal (efeito gnético), do ambiente permanente e do resíduo. Os componentes de variância genética, residual e fenotípica mostraram uma relação positiva, crescente, com o nível de produção e com a classe de desvio-padrão, todavia os coeficientes de herdabilidade não apresentaram a mesma tendência tendo as estimativas mais altas ocorrido no nível baixo de produção e na classe de desvio-padrão médio.
\end{abstract}

Palavras-chave: heterogeneidade das variâncias, níveis de produção, raça Holandesa

\section{Study of the Heterogeneity of the Variances for Productive Characteristics in Holstein Herds in the State of Rio Grande do Sul}

\begin{abstract}
To study heterogeneity of variances and to estimate the heritability coefficients at different levels of herd milk production and at different levels of standard deviation records on 13,053 lactations of 6,828 Holstein cows, sired by 811 bulls and distributed in 78 herds in the State of Rio Grande do Sul from 1988 a 1997 were analyzed. The herds were grouped by milk production and were stratified by the standard deviation of milk production (305 days) in three levels (low, medium and high). The variance components were estimated by Restrict Maximum Likelihood, using the program MTDFREML and a mixed animal model including the fixed effects: class of age, genetic group, herd / nucleus and year-season of calving and the random effects of animal, permanent environmental and residual. The genetic, residual and phenotypic variance components showed a positive relationship with production level and standard deviation class but the heritability coefficients did not present the same trend and the higher estimates occurred at the low production level and at the medium standard deviation class.
\end{abstract}

Key Words: heterogeneity of variance, Holstein breed, production level

\section{Introdução}

O conhecimento de aspectos genéticos de uma população é essencial para a obtenção de informações que venham a orientar os produtores e técnicos na identificação e acasalamento de animais geneticamente superiores, visando um progresso genético maior nos programas de seleção. Para a realização da seleção é preciso ter conhecimento dos valores dos componentes de (co) variância, para que sejam determinados os parâmetros genéticos para as características a serem selecionadas. Da correta estimativa destes parâmetros depende a precisão das estimativas dos valores genéticos e do progresso genético em uma população em processo de seleção.
Valores acurados para estes parâmetros genéticos são necessários para se avaliarem programas de melhoramento, como também para a predição de valores genéticos. Estimativas dos componentes de variância e coeficientes de herdabilidade têm se mostrado heterogêneas de acordo com diferentes níveis de produção, classes de desvio-padrão fenotípico dos rebanhos, tamanho do conjunto de dados além da forma da edição dos dados, dentre outros.

Na maioria dos procedimentos de avaliação genética, utilizando modelos mistos, em gado de leite, simplesmente assumem variâncias genética e residual homogêneas nos diferentes rebanhos. Em alguns trabalhos, no entanto, onde os componentes de

\footnotetext{
${ }^{1}$ Parte da Dissertação de Mestrado do primeiro autor.

2 Mestre em Zootecnia - R. Olavo Bilac, 292/201 - CEP - 97015-440 Santa Maria - RS. E.mail: aemarion@netpar.com.br

${ }^{3}$ Professor do Departamento de Zootecnia - Universidade Federal de Santa Maria, Campus Camobi, CEP - 97105-900 Santa Maria - RS.

E.mail: rorato@ccr.ufsm.br

${ }^{4}$ Aluno de pós-graduação em Zootecnia da Universidade Federal de Santa Maria.
} 
variância para a produção de leite foram estimados em rebanhos agrupados por nível de produção, foi positiva a relação entre nível de produção e as estimativas das variâncias genéticas e residuais (HILL et al., 1983; MIRANDE e VAN VLECK, 1985; LOFGREN et al., 1985; DE VEER e VAN VLECK, 1987; DONG e MAO, 1990; BOLDMAN e FREEMAN, 1990; VALENCIA et al., 1998). Com isso, se as variâncias aumentam com o incremento na média de produção e se assumidas homogêneas, poderão induzir a erros na avaliação genética, podendo os animais serem classificados de forma indevida.

Uma conseqüência direta da heterogeneidade das variâncias em diferentes níveis de produção dos rebanhos é o risco de se selecionar maior proporção de animais de maior variabilidade fenotípica, e não de maior valor genético, podendo, com isso, ocorrer até redução no progresso genético esperado (RAMOS et al., 1996).

Esta heterogeneidade nos componentes de variância reflete diretamente nas estimativas de coeficientes de herdabilidade. HILL et al. (1983), MIRANDE e VAN VLECK (1985), LOFGREN et al. (1985), BOLDMAN e FREEMAN (1990) e DONG e MAO (1990), estudando registros de vacas da raça Holandesa, encontraram tendência de aumento nas herdabilidades com o aumento na média de produção. $\mathrm{O}$ incremento da estimativa de herdabilidade com a elevação do nível de produção ocorre porque as vacas podem expressar melhor o seu potencial genético em rebanhos com altos níveis de produção, devido ao melhor ambiente oferecido com controle de enfermidades e melhor alimentação (HILL et al., 1983). Entretanto, em estudo da heterogeneidade das variâncias em diferentes níveis de produção, RAMOS et al. (1996), utilizando 7.157 lactações de vacas da raça Holandesa, dividiram os rebanhos em nível baixo, médio e alto de produção e estimaram coeficientes de herdabilidade inferiores no nível médio $(0,24$ para a produção de leite-PL e 0,25 para a produção de gordura-PG), seguido do nível alto (0,31 para $\mathrm{PG})$ e do nível baixo (0,38 para PL e 0,35 para PG). RORATO et al. (1998), em estudo similar, encontraram maiores herdabilidades para produção de leite no nível baixo $(0,58)$, seguida pelos níveis alto $(0,32)$ e médio $(0,26)$.

Nas estimativas de componentes de variância para as características produtivas de gado leiteiro também vêm sendo conduzidas investigações sobre a homogeneidade das variâncias em rebanhos agrupados por classes de desvio-padrão, os quais vêm fornecendo considerável evidência da existência da variância hete- rogênea entre as diferentes classes de desvio-padrão.

Autores como HILL et al. (1983) TORRES et al. (1998), COSTA et al. (1999) e TORRES et al. (1999) classificaram os rebanhos pela variância, em vez da média de produção, e observaram que as variâncias genéticas e ambientais apresentaram maiores valores para rebanhos mais variáveis.

LOFGREN et al. (1985) trabalhando com 56.767 observações de vacas da raça Holandesa, primeiramente agruparam os rebanhos por níveis de produção em baixo, médio e alto e estimaram coeficientes de herdabilidade de 0,22, 0,16 e 0,21 , respectivamente, não observando tendência de aumento com o aumento da média. Quando os mesmos rebanhos foram agrupados por classes de desvio-padrão (baixo, médio e alto), estimaram herdabilidades de $0,18,0,19$ e 0,21 , respectivamente, mostrando um incremento com o aumento da variação dentro de rebanho e que os resultados de estimativas de herdabilidade podem diferir de acordo com a média e o desvio-padrão do rebanho.

Em estudo com registros de primeiras lactações de 299.441 vacas da raça Holandesa, SHORT et al. (1990) dividiram os rebanhos em três diferentes classes de desvio-padrão fenotípico de produção de leite e estimaram os coeficientes de herdabilidade de $0,154,0,263$ e 0,226 , respectivamente, para as classes de desvio-padrão baixa, média e alta.

TORRES et al. (1999), em estudo com 109.200 lactações com a finalidade de verificar o efeito da heterogeneidade das variâncias na avaliação genética de vacas da raça Holandesa no Brasil, dividiram os rebanhos em três classes de desvios-padrão, observaram que o coeficiente de herdabilidade para a produção de leite foi maior no nível médio de desviopadrão $(0,28)$, seguido do nível baixo $(0,26)$ e o menor coeficiente foi encontrado no nível alto $(0,25)$.

COSTA (1999) utilizando registros de produção de leite e gordura de primeiras lactações de vacas da raça Holandesa, os quais foram classificados em dois grupos, alto e baixo desvio-padrão fenotípico, encontrou estimativas para os coeficientes de herdabilidade, para as produções de leite e gordura, maiores $(0,30 \mathrm{e}$ 0,20 , respectivamente) nos rebanhos de baixo desviopadrão, em relação aos de alto desvio-padrão $(0,23 \mathrm{e}$ 0,20 , respectivamente).

Este trabalho teve por objetivo verificar o comportamento das estimativas dos componentes de variância e dos coeficientes de herdabilidade para as características produção de leite e de gordura em rebanhos da raça Holandesa agrupados pelo nível de

Rev. bras. zootec., 30(6S):1995-2001, 2001 
produção e em diferentes classes de desvio-padrão fenotípico da produção de leite.

\section{Material e Métodos}

Os registros de produção de leite e gordura utilizados para o desenvolvimento deste estudo, pertencem aos arquivos do Serviço de Controle Leiteiro da Associação de Criadores de Gado Holandês do Rio Grande do Sul (ACGHRS), com sede em Porto Alegre. A propósito de investigar a heterogeneidade das variâncias, utilizaram-se informações de produção de 13.053 lactações, ajustadas para 305 dias, de 4.898 vacas da raça Holandesa paridas entre os anos de 1988 e 1997, filhas de 811 touros e distribuídas em 78 rabanhos pertencentes a 25 núcleos de controle leiteiro (regiões do estado onde o controle leiteiro foi realizado pela mesma pessoa).

Para determinar o comportamento das variâncias em diferentes níveis de produção de leite, os rebanhos foram agrupados conforme o seu nível médio de produção no período estudado. Foram formados três níveis: baixo $(<5.000 \mathrm{~kg})$, médio $(=5.000 \mathrm{~kg} \mathrm{a}<6.000 \mathrm{~kg})$ e alto $(=>6.000 \mathrm{~kg})$, de modo a que cada rebanho só ocorresse em um nível, ficando a composição dos grupos de produção conforme a Tabela 1 .

Para se determinar a heterogeneidade das variâncias em diferentes classes de desvio-padrão, os mesmos rebanhos foram agrupados pelo seu desvio-padrão fenotípico da produção de leite. Foram formados três classes de desvio-padrão: baixo $(<1.400 \mathrm{~kg})$, médio $(=1.400 \mathrm{~kg} \mathrm{a}<1.600 \mathrm{~kg})$ e alto $(=>1.600 \mathrm{~kg})$, de modo que cada rebanho só comparecesse em uma das classe.A formação das classes se deu de maneira a tentar distribuir uniformemente os dados, mas cada classe diferindo em número de observações, rebanhos e touros, ficando a composição das diferentes classes conforme a Tabela 2.

As estimativas dos componentes de variância e coeficientes de herdabilidade, para as características produtivas foram obtidas utilizando-se o Método da Máxima Verossimilhança Restrita - REML, adotandose um modelo animal, incluindo como efeito aleatório o animal (efeito genético), o efeito de ambiente permanente e o resíduo, e como efeitos fixos classe de idade do animal $(1,2, \ldots 12)$, grupo genético $(1=\mathrm{P} 0$ e $2=\mathrm{Grau}$ de sangue igual ou superior a $31 / 32$ da raça Holandesa), rebanho dentro do núcleo de controle leiteiro $(1,2, \ldots, 78)$ e ano-época de parição (época 1 , outubro a março e época 2, abril a setembro). O programa utilizado foi o Multiple Trait Derivative-Free Restricted Maximum Likelihood, MTDFREML, de BOLDMAN et al., 1995. O critério de convergência foi $10^{-6}$.

Omodeloproposto para o estudo em termos matriciais foi: $\mathrm{Y}=\mathrm{X} \beta+\mathrm{Z}_{\mathrm{g}}+\mathrm{W}_{\mathrm{pe}}+\mathrm{e}$; em que: $\mathrm{Y}=$ vetor das variáveis dependentes ( produção de leite

Tabela 1 - Número de lactações, rebanhos, touros e vacas, número médio de observações por rebanho, por touro e por vaca e médias \pm desvios-padrão para as produção de leite e gordura, ajustadas para 305 dias, de acordo com os diferentes níveis de produção de leite

Table 1 - Number of lactations, herds, sires and cows, mean number of observation per herd, sire and cow and mean \pm standard deviation for milk and fat production, adjusted for 305 days, according to different milk production level

\begin{tabular}{|c|c|c|c|}
\hline & \multicolumn{3}{|c|}{$\begin{array}{l}\text { Nível de produção } \\
\text { Production level }\end{array}$} \\
\hline & $\begin{array}{l}\text { Baixo } \\
\text { Low }\end{array}$ & $\begin{array}{l}\text { Médio } \\
\text { Medium }\end{array}$ & $\begin{array}{l}\text { Alto } \\
\text { High }\end{array}$ \\
\hline $\begin{array}{l}\text { Número de lactações } \\
\text { Number of lactations } \\
\text { Rebanhos } \\
\text { Herds }\end{array}$ & 2079 & 6878 & 4096 \\
\hline $\begin{array}{l}\text { Número de rebanhos } \\
\text { Number of herds }\end{array}$ & 15 & 32 & 31 \\
\hline $\begin{array}{l}\text { Média de observações/ } \\
\text { rebanho } \\
\text { Mean oflactations/herd }\end{array}$ & 139 & 215 & 132 \\
\hline Touros & & & \\
\hline $\begin{array}{l}\text { Sires } \\
\quad \text { Número de touros } \\
\quad \text { Number of sires }\end{array}$ & 269 & 470 & 425 \\
\hline $\begin{array}{l}\text { Média de observação/touro } \\
\text { Mean of observation/sires }\end{array}$ & 7,7 & 14,6 & 9,6 \\
\hline Vacas & & & \\
\hline $\begin{array}{l}\text { Cows } \\
\text { Número de vacas } \\
\text { Number of cows }\end{array}$ & 633 & 2013 & 1505 \\
\hline $\begin{array}{l}\text { Número de lactação/vaca } \\
\text { Number oflactation/cow }\end{array}$ & 3,3 & 3,4 & 2,7 \\
\hline $\begin{array}{l}\text { Número de vacas/touro } \\
\text { Number of cows/sire }\end{array}$ & 2,4 & 4,3 & 3,5 \\
\hline Produção de leite $(\mathrm{kg})$ & & & \\
\hline $\begin{array}{l}\text { Milk production }(\mathrm{kg}) \\
\quad \text { Média }\end{array}$ & & & \\
\hline Mean & 4578 & 5489 & 6618 \\
\hline $\begin{array}{l}\text { Desvio-padrão } \\
\text { Standard deviation }\end{array}$ & 1067 & 1504 & 1648 \\
\hline $\begin{array}{l}\text { Produção de gordura ( } \mathrm{kg} \text { ) } \\
\text { Fat production (kg) }\end{array}$ & & & \\
\hline $\begin{array}{l}\text { Média } \\
\text { Mean }\end{array}$ & 150,8 & 177,3 & 221,8 \\
\hline $\begin{array}{l}\text { Desvio-padrão } \\
\text { Standard deviation }\end{array}$ & 42,3 & 58,2 & 58,1 \\
\hline
\end{tabular}


e gordura); $X=$ matriz de incidência associando $\beta$ com $Y ; \beta=$ vetor dos efeitos fixos da classe de idade, da composição genética, do rebanho dentro de época e, como covariável, a duração da lactação; $Z$ = matriz de incidência associando $\mathrm{g}$ com $\mathrm{Y}$; $\mathrm{g}=$ vetor dos efeitos aleatórios do valor genético aditivo direto do touro; $\mathrm{W}=$ matriz de incidência associando pe com $\mathrm{Y}$;

Tabela 2 - Número de observações, rebanhos, touros e vacas, número médio de observações por rebanho, touro e vaca, e médias \pm desvios-padrão para as produções de leite e de gordura, ajustadas para 305 dias, de acordo com as diferentes classes de desvio-padrão fenotípico

Table 2 - Number of observations, herds, sires and cows, mean number of observations by herd, sire and cow and mean \pm standard deviation for milk and fat production, adjusted for 305 days, according to different classes of phenotypic standard deviation

\begin{tabular}{|c|c|c|c|}
\hline & \multicolumn{3}{|c|}{$\begin{array}{l}\text { Classe de desvio-padrão } \\
\text { Class of standard deviation }\end{array}$} \\
\hline & $\begin{array}{l}\text { Baixo } \\
\text { Low }\end{array}$ & $\begin{array}{l}\text { Médio } \\
\text { Medium }\end{array}$ & $\begin{array}{l}\text { Alto } \\
\text { High }\end{array}$ \\
\hline Número de lactações & 3970 & 3989 & 5094 \\
\hline $\begin{array}{l}\text { Number of lactations } \\
\text { Rebanhos } \\
\text { Herds }\end{array}$ & & & \\
\hline $\begin{array}{l}\text { Número de rebanhos } \\
\text { Number of herds }\end{array}$ & 33 & 23 & 22 \\
\hline $\begin{array}{l}\text { Número médio de } \\
\text { observações/rebanho } \\
\text { Average number of } \\
\text { observation/herd }\end{array}$ & 120 & 173 & 232 \\
\hline Touros & & & \\
\hline Sires & & & \\
\hline $\begin{array}{l}\text { Número de touros } \\
\text { Number of sires }\end{array}$ & 436 & 426 & 633 \\
\hline $\begin{array}{l}\text { Média de observações/ } \\
\text { touro } \\
\text { Average number of } \\
\text { observation /sires }\end{array}$ & 9,1 & 9,4 & 8,1 \\
\hline Vacas & & & \\
\hline Cows & & & \\
\hline $\begin{array}{l}\text { Número de vacas } \\
\text { Number of cows }\end{array}$ & 1237 & 1652 & 2259 \\
\hline $\begin{array}{l}\text { Número de lactações/vaca } \\
\text { Number oflactation/cow } \\
\text { Número de vacas/touro }\end{array}$ & a 3,2 & 2,4 & 2,3 \\
\hline $\begin{array}{l}\text { Number of cows/sire } \\
\text { Produção de leite (kg) }\end{array}$ & 2,8 & 3,9 & 3,6 \\
\hline $\begin{array}{l}\text { Milk production }(\mathrm{kg}) \\
\text { Média } \\
\text { Mean }\end{array}$ & 4996 & 6016 & 5997 \\
\hline $\begin{array}{l}\text { Desvio-padrão } \\
\text { Standard deviation }\end{array}$ & 1182 & 1510 & 1879 \\
\hline Produção de gordura (kg) & & & \\
\hline $\begin{array}{l}\text { Fat production }(\mathrm{kg}) \\
\text { Média } \\
\text { Mean }\end{array}$ & 161,9 & 201,9 & 194,9 \\
\hline $\begin{array}{l}\text { Desvio-padrão } \\
\text { Standard deviation }\end{array}$ & 43,5 & 55,4 & 71,1 \\
\hline
\end{tabular}

pe $=$ vetor dos efeitos aleatórios de ambiente permanente do animal; e e $=$ vetor dos efeitos residuais.

\section{Resultados e Discussão}

As estimativas dos componentes de variância genética e de ambiente nos três níveis de produção dos rebanhos, para as características produção de leite e de gordura, estão reunidas na Tabela 3. Observam-se variâncias heterogêneas, crescendo com o aumento do nível de produção. Estes resultados concordam com os relatados por HILL et al. (1983), MIRANDE e VAN VLECK (1985), DE VEER e VAN VLECK (1987), WINKELMAN e SCHAEFFER (1988) e DONG e MAO (1990), os quais observaram uma relação positiva, crescente, entre os níveis de produção de leite dos rebanhos e os componentes de variância.

Os componentes de variância genética e de ambiente para a produção de leite, no nível baixo de produção foram, respectivamente, 61,5 e $42,9 \%$ dos valores estimados para o nível alto, ambos aumentaram com o aumento do nível de produção, tendo a variância de ambiente maior incremento (Tabela 3), reprimindo a variância genética. Para a produção de gordura estas relações foram de 58,5 e 48,7\%, respectivamente, para as variâncias genética e de ambiente, mostrando também, para esta característica, aumento proporcionalmente maior para a variância de ambiente com o aumento do nível de produção.

Os maiores componentes de variância genética, para a produção de gordura, ocorreram nos níveis médio e alto de produção (Tabela 3), possivelmente pelo melhor padrão alimentar adotado nestes níveis o que permitiu que os animais geneticamente superiores para esta característica expressassem este potencial.

Em termos proporcionais, como é demonstrado na Figura 1, observa-se tanto para a produção de leite como para a de gordura maiores variâncias de ambiente e menores variâncias genéticas no nível médio de produção, e as maiores variâncias genética e menores variâncias de ambiente no nível baixo de produção.

As estimativas de herdabilidade, para diferentes níveis de produção de leite e de gordura, estão apresentadas na Tabela 3 . As herdabilidades estimadas para a produção de leite e de gordura não foram homogêneas entre os diferentes níveis de produção dos rebanhos. Os rebanhos do nível médio de produção, foram os que apresentaram o menor coeficiente de herdabilidade, ficando próximo ao do rebanho do

Rev. bras. zootec., 30(6S):1995-2001, 2001 
Tabela 3 - Médias de produção de leite e de gordura \pm desvio-padrão (DP), variância genética $\left(\sigma^{2}\right.$ ), variância de ambiente permanente $\left(\sigma_{\mathrm{pe}}^{2}\right)$, variância de ambiente temporária $\left(\sigma^{2}\right.$ te $)$ e coeficiente de herdabilidade $\left(\mathrm{h}^{2}\right)$ para as características produção de leite e de gordura em diferentes níveis de produção dos rebanhos

Table 3 - Average milk and fat production \pm standard deviation $(D P)$, genetic variance $\left(\sigma^{2}{ }_{\mathrm{a}}\right)$, environmental permanent variance $\left(\sigma^{2}{ }_{p e}\right)$, environmental temporary variance $\left(\sigma^{2}\right.$ te $)$ and heritability coefficients $\left(\mathrm{h}^{2}\right)$ for the characteristics milk and fat production at different level of herd production

\begin{tabular}{|c|c|c|c|c|c|c|}
\hline & \multicolumn{6}{|c|}{$\begin{array}{c}\text { Nível de produção } \\
\text { Production level }\end{array}$} \\
\hline & \multicolumn{2}{|c|}{$\begin{array}{l}\text { Baixo } \\
\text { Low }\end{array}$} & \multicolumn{2}{|c|}{$\begin{array}{c}\text { Médio } \\
\text { Medium } \\
\end{array}$} & \multicolumn{2}{|c|}{$\begin{array}{l}\text { Alto } \\
\text { High }\end{array}$} \\
\hline & $\begin{array}{l}\text { Leite } \\
\text { Milk }\end{array}$ & $\begin{array}{c}\text { Gordura } \\
\text { Fat }\end{array}$ & $\begin{array}{l}\text { Leite } \\
\text { Milk }\end{array}$ & $\begin{array}{c}\text { Gordura } \\
\text { Fat }\end{array}$ & $\begin{array}{l}\text { Leite } \\
\text { Milk }\end{array}$ & $\begin{array}{c}\text { Gordura } \\
\text { Fat }\end{array}$ \\
\hline $\begin{array}{l}\text { Médias }(\mathrm{kg}) \\
\text { Means (kg) }\end{array}$ & 4578 & 150,8 & 5489 & 177,2 & 6618 & 221,8 \\
\hline $\mathrm{DP}(\mathrm{kg})$ & 1067 & 42,3 & 1504 & 58,2 & 1648 & 58,1 \\
\hline$\sigma_{\mathrm{a}}^{2}\left(\mathrm{~kg}^{2}\right)$ & 189.641 & 212,2 & 273.925 & 370 & 308.213 & 362,7 \\
\hline$\sigma_{\mathrm{pe}}^{2}\left(\mathrm{~kg}^{2}\right)$ & 2.072 & 0,54 & 6.162 & 11,24 & 6.177 & 28,2 \\
\hline$\sigma_{\text {te }}^{2}\left(\mathrm{~kg}^{2}\right)$ & 627.154 & 823,1 & 1.337 .200 & $2.047,3$ & 1.460 .872 & 1689,4 \\
\hline $\mathrm{h}^{2}$ & 0,23 & 0,20 & 0,16 & 0,15 & 0,17 & 0,17 \\
\hline
\end{tabular}

nível de produção alto, tendo os rebanhos do nível baixo de produção os maiores coeficientes de herdabilidade, concordando com os resultados obtidos por RAMOS et al. (1996) e RORATO et al. (1998). Entretanto, estes resultados discordam daqueles relatados por MIRANDE e VAN VLECK (1985), DE VEER e VAN VLECK (1987) e BOLDMAN e FREEMAN (1990), os quais observaram maiores coeficientes de herdabilidade com o aumento da média de produção dos rebanhos.

O comportamento dos coeficientes de herdabilidade neste trabalho, acompanhou a variação dos componentes de variância, os quais aumentaram com o aumento do nível de produção, todavia, o aumento da variância de ambiente foi proporcionalmente maior. A variância genética foi proporcionalmente maior no nível baixo e menor no nível médio de produção (Figura 1), ou seja, com o aumento do nível de produção do rebanho ocorreu uma compressão da variância genética pela variância de ambiente, induzindo a menores coeficientes de herdabilidade no nível médio e a maiores no nível baixo de produção.

Os componentes de variância genética e de ambiente em diferentes classes de desvio-padrão, para as características produção de leite e gordura, estão apresentados na Tabela 4. Observa-se que as variâncias genéticas elevam-se com o aumento do desvio-padrão médio tanto para a produção de leite quanto para a produção de gordura. Vários trabalhos têm mostrado maiores variâncias genéticas e de ambiente, à medida que aumenta o desvio-padrão médio da classe (HILL et al., 1983; SHORT et al., 1990; VALÊNCIA et al., 1998; TORRES et al., 1999; e COSTA et al., 1999).

A classe de desvio-padrão médio, quando comparada

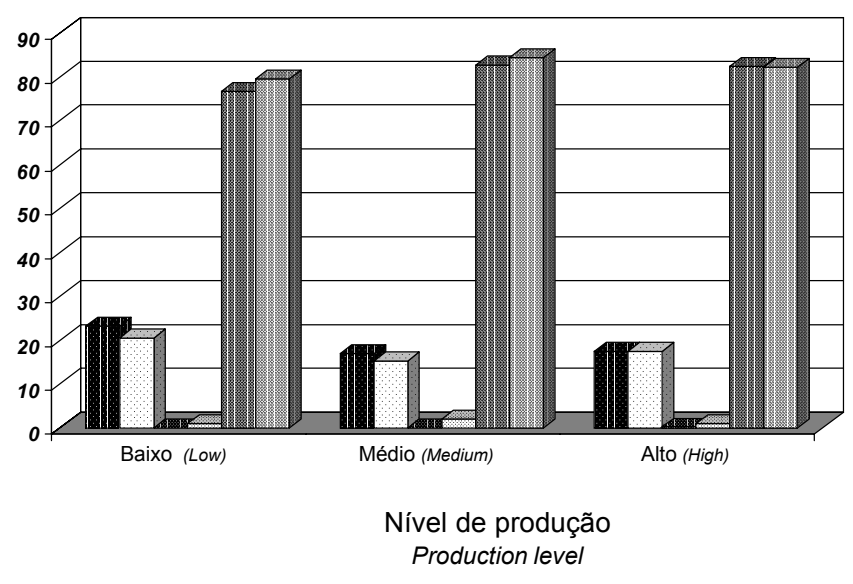

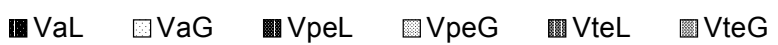

Figura 1 - Porcentagem de variância genética (VaL e VaG), de ambiente permanente (Vpel e VpeG) e ambiente temporário (VteL e VteG), para produção de leite (L) e gordura (G), em diferentes níveis de produção nos rebanhos.

Figure 1 - Percentage of genetic ( $V a L$ and $V a G)$, permanent environmental (VpeL and VpeG) and temporary environmental variance (VteL and VteG), for milk $(M)$ and fat $(F)$ production, at different level of herd production. 
Tabela 4 - Médias de produção de leite e de gordura \pm desvio-padrão (DP), variância genética $\left(\sigma^{2}{ }_{a}\right)$, variância de ambiente permanente $\left(\sigma_{\text {pe }}^{2}\right)$, variância de ambiente temporário $\left(\sigma_{\text {te }}^{2}\right)$ e coeficiente de herdabilidade $\left(h^{2}\right)$ para produção de leite e gordura em diferentes classes de desvio-padrão fenotípico

Table 4 - Average milk and fat production \pm standard deviation $(S D)$, genetic variance $\left(\sigma^{2}{ }_{a}\right)$, permanent environmental variance $\left(\sigma^{2}\right.$ pe), temporary environment variance $\left(\sigma^{2}\right.$ te $)$ and heritability coefficient $\left(h^{2}\right)$ for milk and fat production at different class of phenotypic standard deviation

\begin{tabular}{|c|c|c|c|c|c|c|}
\hline & \multicolumn{6}{|c|}{$\begin{array}{l}\text { Classes de desvio-padrão } \\
\text { Class of standard deviation }\end{array}$} \\
\hline & \multicolumn{2}{|c|}{$\begin{array}{l}\text { Baixo } \\
\text { Low }\end{array}$} & \multicolumn{2}{|c|}{$\begin{array}{c}\text { Médio } \\
\text { Medium }\end{array}$} & \multicolumn{2}{|c|}{$\begin{array}{l}\text { Alto } \\
\text { High }\end{array}$} \\
\hline & $\begin{array}{l}\text { Leite } \\
\text { Milk }\end{array}$ & $\begin{array}{c}\text { Gordura } \\
\text { Fat }\end{array}$ & $\begin{array}{l}\text { Leite } \\
\text { Milk }\end{array}$ & $\begin{array}{c}\text { Gordura } \\
\text { Fat }\end{array}$ & $\begin{array}{l}\text { Leite } \\
\text { Milk }\end{array}$ & $\begin{array}{c}\text { Gordura } \\
\text { Fat }\end{array}$ \\
\hline $\begin{array}{l}\text { Médias (kg) } \\
\text { Means }\end{array}$ & 4996 & 161,9 & 6016 & 201,9 & 5997 & 194,9 \\
\hline D P (kg) & 1182 & 43,5 & 1510 & 55,4 & 1879 & 71,1 \\
\hline$\sigma_{\mathrm{a}}^{2}\left(\mathrm{~kg}^{2}\right)$ & 167.636 & 189,9 & 306.960 & 340,6 & 366.308 & 415,1 \\
\hline$\sigma_{\text {pe }}^{2}\left(\mathrm{~kg}^{2}\right)$ & 14.718 & 0,61 & 10.256 & 46,4 & 12,3 & 0,001 \\
\hline$\sigma_{\text {te }}^{2}\left(\mathrm{~kg}^{2}\right)$ & 681.498 & 896,1 & 1.203 .778 & $1.514,9$ & 1.671 .904 & $2.430,9$ \\
\hline $\mathrm{h}^{2}$ & 0,19 & 0,17 & 0,20 & 0,18 & 0,18 & 0,15 \\
\hline
\end{tabular}

com a de desvio-padrão baixo, revelou variâncias genética e de ambiente temporário maiores, respectivamente, em 1,83 e 1,76 vezes para a produção de leite e 1,79 e 1,69 vezes para a produção de gordura, mostrando aumento relativamente maior para a variância genética do que para a variância de ambiente. Isto deve ter ocorrido, provavelmente, em função de os animais terem tido maior possibilidade de expressarem o seus potenciais genéticos na classe de desvio-padrão médio. Ao compararem-se os componentes de variância entre a classe de desviopadrão médio a de desvio-padrão alto, observa-se aumento na variância genética e de ambiente respectivamente de 1,19 e 1,39 vezes para a produção de leite e 1,22 e 1,60 para a produção de gordura, verificando-se maior aumento relativo na variância de ambiente. Na Figura 2, na qual estão apresentados os componentes de variância em termos proporcionais, observa-se que a variância de ambiente aumentou e a genética diminuiu com o aumento do desvio-padrão, sendo este comportamento mais acentuado entre as classes de desvio-padrão médio e alto, sugerindo a compressão da variância genética.

Ao se agruparem os rebanhos em classes de desvio-padrão, observam-se as maiores médias de produção, tanto para leite quanto para gordura, na classe de desvio-padrão médio, todavia as maiores variâncias ambiental e genética ocorreram na classe de desvio-padrão alto sugerindo que os componentes de variância crescem com o desvio-padrão médio dos rebanhos (Tabela 4). No entanto, a variância de ambiente permanente não demonstrou o mesmo com- portamento, apresentando menores valores com o aumento do coeficiente de variação das classes.

Os coeficientes de herdabilidade estimados nas diferentes classes de desvio-padrão para as características estudadas estão apresentados na Tabela 4. Observa-se que as maiores estimativas ocorreram na classe de desvio-padrão médio, seguida pelas de des-

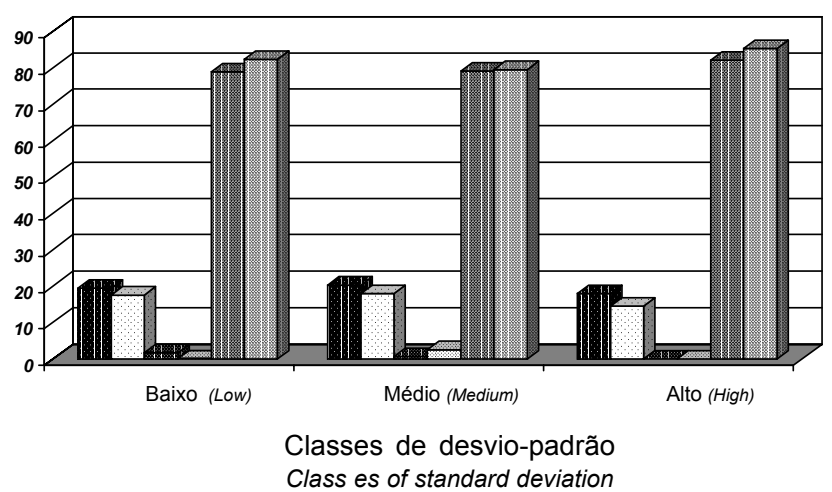

VaL $\square$ VaG Vpel $\square$ VpeG 圆Vtel $\quad$ V VteG

Figura 2 - Porcentagem de variância genética (VaL e VaG), de ambiente permanente (Vpel e VpeG) e de ambiente temporário (VteL e VteG), para produção de leite $(L)$ e de gordura $(G)$, em diferentes classes de desvio-padrão.

Figure 2 - Percentage of genetic (VaL and VaG), permanent environmental (VpeL and $V p e G$ ) and temporary environmental variances (VteL and VteG), for milk (M) and fat $(F)$ production, at different class of standard deviation. 
vio-padrão baixo e alto. Esta maior estimativa para a classe de desvio-padrão médio sugere que a expressão do genótipo dos touros no desempenho de sua progênie será maior na classe de desvio-padrão médio do que nas de desvio-padrão baixo e alto. Neste caso os coeficientes de herdabilidade não se comportaram da mesma maneira que ao se agrupar pelas médias de produção, semelhantemente ao relatado por HILL et al. (1983) e LOFGREN et al. (1985). Estes resultados também concordam com os relatos de SHORT et al. (1990) e TORRES et al. (1999), que encontraram maiores coeficientes de herdabilidade em rebanhos de desvio-padrão médio, entretanto discordam daqueles verificados por COSTA et al. (1999), os quais encontraram maiores coeficientes de herdabilidade em rebanhos de desvio-padrão baixo.

\section{Conclusões}

Os componentes de variâncias heterogêneos nos diferentes níveis de produção e classes de desviopadrão contrariam a suposição de variâncias homogêneas e, neste caso, poderão mascarar a avaliação genética, diminuindo a sua acurácia e, conseqüentemente, a eficiência da seleção, principalmente de vacas que são geralmente avaliadas dentro de um mesmo rebanho. Para os touros isto é parcialmente contornado pela utilização da inseminação artificial, a qual proporciona a avaliação das progênies em diferentes rebanhos.

A heterogeneidade observada nos coeficientes de herdabilidade nos diferentes níveis de produção e classes de desvio-padrão revela que as respostas esperadas pela seleção também serão diferentes para os diferentes níveis de produção ou classes de desvio-padrão, o que deve ser considerado nos programas de melhoramento.

\section{Agradecimento}

Ao CNPq, pela bolsa de Mestrado.

À Associação de Criadores de Gado Holandês do Rio Grande do Sul (ACGHRS), pela cessão dos dados que permitiram a realização deste trabalho.

\section{Referências Bibliográficas}

BOLDMAN, K.G., FREEMAN, A.E. 1990. Adjustment for heterogeneity of variances by herd production level in dairy cow and sire evaluation. J. Dairy Sci., 73(2):503-512.

BOLDMAN, K.G., KRIESE, L.A., VAN VLECK, L.D et al. 1995. A manual for use of MTDFREML - A set of programs to obtain estimates of variances and covariances(DRAFT), Lincoln: Department of Agriculture/Agricultural Research Service. 120p.

COSTA, C.N. Implicação da heterogeneidade de variância da produção de leite em rebanhos de bovinos da raça Holandesa no Brasil. In: II SIMPÓSIO NACIONAL DA SOCIEDADE BRASILEIRA DE MELHORAMENTO ANIMAL, 1998, Uberaba, MG. Anais... Uberaba, 1998. p.397-400.

COSTA, C.N. 1999. An investigation into heterogeneity of variance for milk and fat yield of Holstein cows in brasilian hard environments. Genetics and molecular biology, 22(3):375-381.

DE VEER, J.C., VAN VLECK, L.D. 1987. Genetic parameters for first lactation milk yields at three levels of herd production. J. Dairy Sci., 70(2):1434-1441.

DONG, M.C., MAO, I.L. 1990. Heterogeneity of (co)variance and heritability an different levels of intra herd milk production variance and of herd average. J. Dairy Sci., 73(3):843-851.

HILL, W.G., EDWARDS, R.M., AHMED, M.K.A. et al. 1983. Heritability of milk yield and composition at different levels and variability of production. Anim. Prod., 36(1):59- 68.

LOFGREN, D.L., VINSON, W.E., PEARSON, R.E. et al. 1985. Herdability of milk yield at different herd means and variance for production. J. Dairy Sci., 68(10):2737-2739.

MIRANDE, S.L., VAN VLECK, L.D. 1985. Trends in genetic and phenotypic variances for milk production. J. Dairy Sci., 68(9):2278-2286.

RAMOS, A.A., VALENCIA, E.F.T., WECHSLER, F.S. et al. Heterogeinidade da variância das características produtivas de bovinos da raça Holandesa nos trópicos. I - Estratificação por nível de produção de rebanho. In: XXXIII REUNIÃO ANUAL DA SOCIEDADE BRASILEIRA DE ZOOTECNIA, 1996, Fortaleza, CE, Anais... Fortaleza, 1996:71-73.

RORATO, P.R.N., VAN VLECK, D., VERNEQUE, R. et al. Interação genótipo-ambiente para a produção de leite em rebanhos da raça Holandesa no Brasil. (II) Utilizando o modelo animal. In: REUNIÃO ANUAL DA SOCIEDADE BRASILEIRA DE ZOOTECNIA, 35., 1998, Botucatu, SP. Anais... Botucatu, 1998. p.395-397.

SHORT, T.H., BLAKE, R.W., QUAAS, R.L. et al. 1990. Heterogeneous within-herd variance. 1 - Genetic parameters for first and second lactation milk yields of grade Holstein cows. J. Dairy Sci., 73(11):3312-3320.

VAlENTE, J., PENNA, V.M., TORRES FILHO, R.A. Heterogeneidade de variância na avaliação genética de bovinos da raça Holandesa no Brasil, In: REUNIÃO ANUAL DA SOCIEDADE BRASILEIRA DE ZOOTECNIA, 35., 1998, Botucatu, SP. Anais... Botucatu, 1998. p.398-400.

TORRES , R.A., BERGMANN, J.A.G., COSTA, C.N. et al. 1998. Hetetogeneidade de variância na avaliação genética em bovinos da raça Holandesa no Brasil. In: REUNIÃO ANUAL DA SOCIEDADE BRASILEIRA DE ZOOTECNIA, 35., 1998, Botucatu, SP. Anais... Botucatu, 1998. p.398-400.

VALENCIA, E.T., RAMOS, A.A., WESCHLER, F.S. et al. Heterogeneidade dos componentes de variância, na produção de leite de rebanhos dos Estados de São Paulo e Paraná, Brasil, In: REUNIÃO ANUAL DA SOCIEDADE BRASILEIRA DE ZOOTECNIA, 35., 1998, Botucatu, SP. Anais... Botucatu, 1998. p.488-490.

WINKELMAN, A., SCHAEFFER, L.R. 1988. Effect of heterogeneity of variance on dairy sire evaluation. J. Dairy Sci., 71(11):3033-3039.

Recebido em: $16 / 11 / 00$ Aceito em: 27/06/01 